\title{
The Neural Correlates of Subjective Utility of Monetary Outcome and Probability Weight in Economic and in Motor Decision under Risk
}

\author{
Shih-Wei Wu, ${ }^{1}$ Mauricio R. Delgado, ${ }^{2}$ and Laurence T. Maloney ${ }^{3,4}$ \\ ${ }^{1}$ Institute of Neuroscience, National Yang-Ming University, Taipei 112, Taiwan, ${ }^{2}$ Department of Psychology, Rutgers University, Piscataway, New Jersey \\ 08854, and ${ }^{3}$ Department of Psychology and ${ }^{4}$ Center for Neural Science, New York University, New York, New York 10003
}

In decision under risk, people choose between lotteries that contain a list of potential outcomes paired with their probabilities of occurrence. We previously developed a method for translating such lotteries to mathematically equivalent "motor lotteries." The probability of each outcome in a motor lottery is determined by the subject's noise in executing a movement. In this study, we used functional magnetic resonance imaging in humans to compare the neural correlates of monetary outcome and probability in classical lottery tasks in which information about probability was explicitly communicated to the subjects and in mathematically equivalent motor lottery tasks in which probability was implicit in the subjects' own motor noise. We found that activity in the medial prefrontal cortex (mPFC) and the posterior cingulate cortex quantitatively represent the subjective utility of monetary outcome in both tasks. For probability, we found that the mPFC significantly tracked the distortion of such information in both tasks. Specifically, activity in mPFC represents probability information but not the physical properties of the stimuli correlated with this information. Together, the results demonstrate that mPFC represents probability from two distinct forms of decision under risk.

\section{Introduction}

A choice among possible actions can be modeled as a choice among lotteries. A lottery is a list of potential outcomes $O_{i}, i=1$, $\ldots, n$ with associated probabilities of occurrence $p_{i}, i=1, \ldots, n$. In an economic decision task, the decision maker may be asked to decide between $(0.05, \$ 2000 ; 0.95,0)$, a $5 \%$ chance of getting $\$ 2000$ and otherwise nothing, and $(0.5, \$ 200 ; 0.5,0)$, a $50 \%$ chance of winning $\$ 200$ or nothing. A decision maker who knows the probabilities associated with outcomes is engaged in decision under risk (Knight, 1921).

Research on the neural substrates of decision making has focused on such "classical" tasks: subjects choose between lotteries with monetary outcomes and probabilities explicitly specified in numerical or graphical form (Huettel et al., 2006; Paulus and Frank, 2006; Berns et al., 2008; Tobler et al., 2008; Christopoulos et al., 2009; Hsu et al., 2009), yet many of the decisions we face every day involve choices between actions whose outcomes are uncertain only because of perceptual and motor "noise" (Newsome et al., 1989; Trommershäuser et al., 2003a,b; Heekeren et

\footnotetext{
Received Jan. 31, 2011; revised April 23, 2011; accepted April 27, 2011.

Author contributions: S.-W.W., M.R.D., and L.T.M. designed research; S.-W.W. performed research; S.-W.W., M.R.D., and L.T.M. analyzed data; S.-W.W., M.R.D., and L.T.M. wrote the paper.

This work was supported by National Science Council Grant NSC 99-2410-H-010-013-MY2 (S.-W.W.), National Institutes of Health Grants DA027764 (M.R.D.) and EY019889 (L.T.M.), and a Humboldt Research Award (L.T.M.). We thank the reviewers for helpful comments and suggestions. We also thank Yu-Hsin Hsiao for assistance on data collection. We acknowledge MRI support from National Yang-Ming University, Taiwan, which is in part supported by the Ministry of Education plan for the Top University Program.

Correspondence should be addressed to Shih-Wei Wu, Institute of Neuroscience, National Yang-Ming University, Number 155, Section 2, Linong Street, Taipei 112, Taiwan. E-mail: swwu@ym.edu.tw.

DOI:10.1523/JNEUROSCI.0540-11.2011

Copyright $\odot 2011$ the authors $\quad 0270-6474 / 11 / 318822-10 \$ 15.00 / 0$
}

al., 2004; Körding and Wolpert, 2004; Churchland et al., 2006). In these situations, probability information is not explicitly given and has to be estimated based on the knowledge of the noise properties in the environment or within the neural system. The neural representation of this kind of probability information, particularly in the motor domain, is not well characterized.

We recently developed methods (see Materials and Methods) that permit comparison of economic decisions under risk and analogous movement tasks (Wu et al., 2009). We designed simple motor tasks equivalent to lotteries and asked subjects to choose between these "motor lotteries" as they might choose between lotteries in economic tasks. Of critical importance, information about probability was explicitly provided to the subjects in the economic lottery tasks but not in motor lottery tasks. Instead, probability was implicit in the subjects' own motor noise.

Using functional magnetic resonance imaging, we seek to address the following questions. What are the neural correlates of probability when it is the result of the subjects' own motor noise? How might the neural correlates underlying the processing of potential outcome and probability information differ between economic and motor tasks? In what brain areas might probability of different sources converge and be commonly represented?

We found evidence for a common representation of potential outcome and probability in the medial prefrontal cortex (mPFC) in the classical economic lottery task and in the motor lottery task. Furthermore, activity in mPFC quantitatively tracked the subjective utility of outcome and the distortion of probability information inferred from the subjects' choice behavior. Although many studies have shown that mPFC encodes the value of different categories of goods (Plassmann et al., 2007; Chib et al., 
A

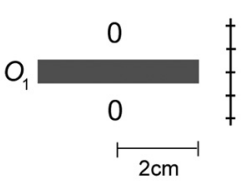

C Classical

$(0.5, \$ 200 ; 0.5, \$ 0)$

Motor $\$ 0$

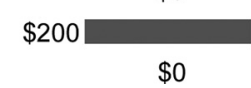

$\$ 0$

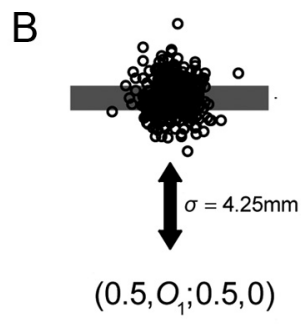

$(0.05, \$ 2000 ; 0.5, \$ 0)$

$\sigma=4.25 \mathrm{~mm}$

or $\frac{\$ 0}{\$ 0} \$ 2000$

Figure 1. Construction of a motor lottery. $A, A$ single target of a rapid pointing task. The subjects rapidly attempted to hit such targets with the index fingers of their dominant hand. $\boldsymbol{B}$, A total of 355 movement endpoints for one subject are superimposed. The distribution is close to isotropic bivariate Gaussian with $\sigma=4.25 \mathrm{~mm}$. The height of the target was adjusted so that this subject had a $50 \%$ chance of hitting the target and earning a reward $0_{1}$. The resulting motor lottery was equivalent to a lottery $(0.5,0 ; 0.5,0)$. C, In a later session, the subjects were shown either pairs of classical lotteries or pairs of motor lotteries and were simply asked to choose the one they preferred. A pair of classical lotteries in which information about monetary outcomes and their probabilities were explicitly stated and a pair of equivalent motor lotteries in which probabilities were implicit in the subjects' own motor noise are shown. The chosen lottery was not executed after subjects made their choices and hence no feedback on winning was available during the decision-making session.

2009), no study to date has shown that mPFC encodes different sources of probability information in decision under risk, especially in situations in which probability had to be estimated based on motor noise.

\section{Materials and Methods}

\section{Overall design}

There were four sessions in the experiment. The first session was a control task in which subjects judged either the size of rectangles or magnitude of numbers. The second was a motor training session: a single session in which the subjects were trained to perform a rapid pointing task. The third session was a decision-making session consisting of choices between motor lotteries and choices between classical lotteries. In the last session, subjects estimated their probability of success in the motor task.

\section{The rapid pointing task}

During the motor training session, subjects were trained to reach and touch a rectangular target presented on a touch screen with their index finger, within $300 \mathrm{~ms}$ (Fig. $1 \mathrm{~A}$ ). Subjects won $O_{1}$ if they hit the target within the time limit, and otherwise 0 if they hit anywhere else on the screen or exceeded the time limit. For more details on the payoff structure for this rapid pointing task, see Procedure below.

Because of the very limited time window, the realization of such speeded movement was stochastic in nature. The probability of hitting the target and hence winning $O_{1}$ depended on the noise intrinsic in the motor system. In past work and in the experiment presented here (Trommershäuser et al., 2003a,b, 2005; Wu et al., 2006, 2009), we found that movement endpoints were well described by an isotropic bivariate Gaussian distribution. We used the SD of the movement endpoints, $\sigma$, to characterize motor noise and we estimated each subject's $\sigma$ separately. To obtain a reliable measure of $\sigma$, we trained subjects extensively at hitting a single target that varied in size from trial to trial. In Figure $1 B$, we superimposed 355 movement endpoints from an actual subject with $\sigma=4.25$ $\mathrm{mm}$. Subjects could maximize their expected gain only by aiming at the

center of the rectangle. In past work and in the current study, we verified that any biases in aiming did not affect our conclusions.

\section{Constructing a motor lottery}

Once we have an accurate estimate of $\sigma$ for a particular subject, we can adjust the height of the target so that the subject's probability of hitting it has any specified value. To distinguish the lottery in motor task from the classical lottery, we referred to the motor task as the motor lottery. The parameter $\sigma$ is all that is needed to translate a classical lottery to a motor lottery. The relationship between a lottery $(p, \$ x ; 1-p, \$ 0)$ and the height $(h)$ of the equivalent motor lottery is the following:

$$
p=\int_{y_{0}-1 / 2 h}^{y_{0}+1 / 2 h} \frac{1}{\sqrt{2 \pi} \sigma} e^{-\frac{\left(y-y_{0}\right)^{2}}{2 \sigma^{2}}} d y,
$$

where $y$ represents the vertical axis, and $y_{0}$ is the center of the motor target.

We chose the target in Figure $1 B$ so that the subject whose endpoints are shown has a $50 \%$ chance of hitting it. The resulting motor lottery was equivalent to a lottery $\left(0.5, O_{1} ; 0.5,0\right)$ for this subject.

In the decision-making session, we presented pairs of classical lotteries or pairs of equivalent motor lotteries to the subjects and asked them to choose the one they preferred on every trial. In Figure $1 C$, we showed a pair of classical lotteries, $(0.5,200 \mathrm{NTD})$ and $(0.05,2000$ NTD) (in which NTD is the National Taiwan Dollar; US $\$ 1=30$ NTD), and an equivalent pair of motor lotteries tailored to a subject with motor noise $\sigma=4.25 \mathrm{~mm}$.

We emphasize that no chosen lottery was realized during the decisionmaking session. The subjects only made a button-press movement to indicate which lottery from a pair of lotteries she preferred. She did so during both classical and motor lottery tasks and at the same time window within a trial.

The subjects were told that, at the end of the experiment, two of their chosen options, one from the classical pairs and one from the motor pairs would be selected at random and executed. They would receive the outcomes of these two lotteries as a bonus (see below, Payoffs). Subjects could potentially win up to 4000 NTD bonus (\$133), although their actual winnings were likely to be less.

\section{Subjects}

Sixteen right-handed subjects participated in the experiment (11 women; mean age, 23 years). One subjects' fMRI data were excluded from additional fMRI analysis because of excessive head motion during the scans. Before the experiment, all participants gave informed consent in accordance with the procedures of the University Committee on Activities Involving Human Subjects of National Yang-Ming University, Taiwan. The subjects were paid 400 NTD for their participation of the experiment and earned an average bonus of 858 NTD from the motor training session and playing the lotteries (bonus range, 279-2594 NTD).

\section{Procedure}

Session 1: size judgment task. This session was conducted inside the MRI scanner. The goal of this session was to serve as a control comparison across the motor and classical lottery tasks with respect to the physical properties of the stimuli. In a size judgment task, the subjects were asked to compare either the size of two numbers or the size of two rectangular targets. In every trial, two options were presented, with each option consisting of a single number and a rectangular target. The number and the target size of one of the options remained fixed within a block of trials. This option was termed the reference option. The other option, whose number and target size changed from trial to trial, was called the varying option. An instructional colored cue also appeared at the center of the screen during a trial, with the particular cue color indicating which component of the options, number or rectangular target, to compare. When the cue was green, the subjects were asked to compare the size of the targets. Alternatively, the subjects were asked to compare the size of the numbers when the cue was red. There were three blocks of trials, each consisting of 24 trials. Each block had a unique reference option. Before 
the start of a block, the subjects were briefly shown the reference item for that block.

We emphasize that the stimuli in this task were identical to the motor lottery task that took place in session 3 , only that the subjects had no knowledge of the similarity between them. During this session, subjects were not aware of the subsequent sessions to come. Because we had no information about the subjects' motor noise at this point of the experiment, we cannot create rectangular targets of the same sizes as those in the later motor lottery task. We approximated the target size to that in the motor lottery task by using the mean motor noise of 10 pilot subjects.

Session 2: motor training. This session was conducted in a behavioral testing room. The goals of this session were to allow the subject to practice the rapid pointing task (described previously) and to allow the experimenter to reliably estimate each subject's motor noise, $\sigma$, the SD of movement endpoints. During motor training, subjects performed rapid pointing to targets presented on a touch screen. The targets carried small monetary rewards of 100 points $(100$ points $=1$ NTD $)$. On every trial, the subject had only $300 \mathrm{~ms}$ to hit the target. If she hit the target, she won 100 points, 0 if anywhere else on the screen was hit, and lost 700 points if the finger did not reach the touch screen within $300 \mathrm{~ms}$. We implemented the monetary penalty simply for the purpose of training the subjects to reach and attempt the target within the time limit. In past and current experiments, the number of trials the subjects did not reach the touch screen within $300 \mathrm{~ms}$ and hence received penalty was usually below $3 \%$ of the total number of trials. We varied the size of the target (six different sizes) from trial to trial. There were 12 blocks of 30 trials each. In each block, we randomized the order in which the different targets were presented. Each target size was repeated five times in each block. During session 2, subjects were not aware of the subsequent decision-making session to come.

Session 3: decision making under risk. This session was conducted inside the MRI scanner. The goals of this session were to compare choice performance and BOLD activity between classical decision under risk task and an equivalent motor task. In every trial, subjects were presented with either a pair of motor lotteries or a pair of classical lotteries and had to choose the one she preferred. The lotteries were presented for $4 \mathrm{~s}$ and were followed by a fixation period $(2 \mathrm{~s})$. After fixation, a yellow dot appeared at the center of the screen for $2 \mathrm{~s}$, during which the subjects had to indicate her choice by pressing either the left or right button. The intertrial interval was $12 \mathrm{~s}$. For details on the trial sequence of the task, see Figure 2, $A$ and $B$.

The motor lottery task and the classical lottery task were run in separate blocks of trials. There were three blocks of classical lottery task and three blocks of equivalent motor lottery task. The order of motor and classical blocks was randomized for each subject. Each block consisted of 24 trials. A single trial consisted of a pair of lotteries. One of the lotteries remained fixed within a block and was termed the reference lottery, whereas the outcome and probability of the other lottery varied from trial to trial and hence was called the varying lottery.

The varying lotteries were determined by the outcome-probability matrix shown in Figure 2C. There were four levels of outcomes, ranging from 100 to 2000 NTD. There were four levels of probability ranging from 0.05 to 0.95 . The color of each cell in the matrix was scaled according to its expected value (EV). Note that the diagonal entries all had the same EV of 100 NTD. There were three possible reference lotteries, with each block having a fixed reference lottery. All the reference lotteries had the same EV of 100 NTD. For the varying lotteries, in a block of trials, each diagonal entry had three repetitions, while each of the remaining 12 off-diagonal entries was placed in a single trial. Thus, a single block had 24 trials, with half of the trials consisting of lottery pairs having the same EV. This design ensured that, at least in half of the trials within a block, the decision would not be trivial. Meanwhile, the correlation between outcome value and probability for the varying lottery within a block was kept at a relatively low value at $\sim 0.3$, which would allow us to construct the general linear model (GLM) for fMRI data analysis by including both outcome value and probability as regressors without introducing high degree of collinearity between the two in the GLM.

Subjects indicated whether she preferred the varying lottery or the reference lottery by pressing one of two buttons with their left or right
A

\section{Classical lottery task}

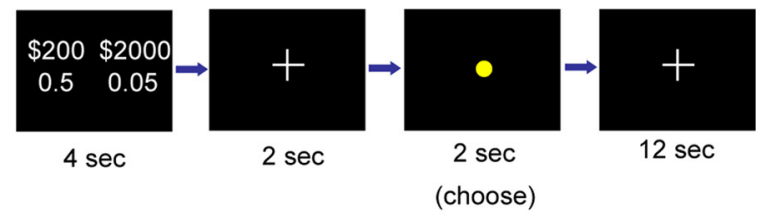

B

Motor lottery task

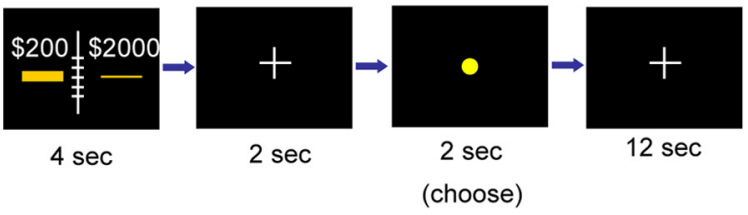

C

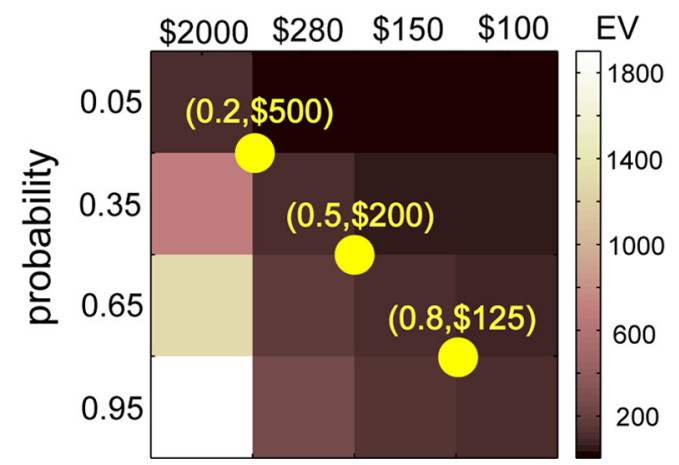

Figure 2. Experimental design. $\boldsymbol{A}$, Trial sequence of the classical lottery task. A pair of classical lotteries presented for $4 \mathrm{~s}$ and conveying information about outcome and probability. A short fixation ( $2 \mathrm{~s}$ ) followed the lottery presentation period. After the fixation, a yellow dot appeared on the screen for $2 \mathrm{~s}$, during which the subjects had to indicate her choice by pressing one of two buttons. In particular, the subjects had to indicate whether she preferred the varying lottery to the reference lottery or not. The intertrial interval was $12 \mathrm{~s}$. B. Trial sequence of the motor lottery task. The trial sequence was the same as the classical lottery task, and the subjects' task was the same: to choose between motor lotteries. A ruler was displayed at the center of the screen to serve as a reference to the subjects in judging the size of the targets attributable to difference in viewing conditions between the motor training session and the fMRI session. In both the classical lottery task and the motor lottery task, no chosen lottery was realized during the fMRI session. C, Lottery design. Every pair of lotteries consisted of a reference lottery and a varying lottery. The varying lottery was selected from the $4 \times 4$ outcome-probability matrix shown here. The color map represented the EV of the lotteries. There were three possible reference lotteries indicated by the yellow dots on the matrix. The diagonal entries of the matrix, and the reference lotteries, all had the same EV. The values are in NTD. 30 NTD is approximately US $\$ 1$.

thumb. For half of the subjects, the button corresponding to the right thumb was designated for the varying lottery, whereas the left thumb indicated choosing the reference lottery. For the other half of the subjects, the finger-button correspondence was reversed.

We also emphasized to the subjects that their chosen lotteries would not be realized during the session. They were informed that, after the conclusion of the session, two of the lotteries they chose, one from the motor trials and one from the classical trials, would be selected at random and executed. The amount they won from this session would be part of their final payoff.

Note that, in both the motor training session and this session, we presented a "ruler" next to the motor targets (Figs. 1 A, 2B) to control for possible differences in viewing conditions between the two sessions (the motor training session was conducted in the behavioral testing room, this session was conducted inside the MRI scanner). During the training session, the subjects were instructed to pay attention to the size of the 
targets relative to the ruler, but we emphasized that the ruler played no role in deciding whether a target was hit or not. During the decisionmaking session, we presented the ruler in the motor lottery task. We instructed the subjects that the same ruler she saw in the training session was again presented and that she should evaluate the targets and their corresponding probabilities of hit with respect to the ruler. That is, a target that occupied the same area on the ruler in the fMRI session would occupy the same area in the behavioral session, although the perception of size of the same target might be different between the two sessions as a result of differences in viewing conditions. We chose not to control for visual angle of the target because there is often an effect of size constancy and therefore, although the retinal size of the target would be controlled when visual angle was controlled, the perception of target size may still be different and hence the estimation of the probability of hit. We asked the subjects to give verbal estimates of the probability of hit at different target sizes in both the behavioral testing room and inside the MRI scanner to verify that such manipulation was effective in the sense that the verbal estimate of the same target did not differ across these two sessions.

Session 4: estimation. After the conclusion of session 3, the subjects were asked to provide verbal estimate of the probability of hitting the target while still inside the scanner. The subjects were presented with five different target sizes, each repeating twice. The five different targets were constructed for each subject separately by taking into account her motor noise such that the probability of hit corresponded to $0.05,0.25,0.5,0.75$, and 0.95 . After the subject gave her verbal estimates, she was taken back to the behavioral testing room. The subject was then presented with the same targets and again was asked to give verbal estimates.

\section{Payoffs}

After giving verbal estimates, two lotteries were selected at random, one from the motor lotteries the subjects chose and the other from the chosen classical lotteries. To select the lotteries from each domain (motor or classical), subjects first picked one poker chip out of an urn that contained numbered chips equal to the number of the trials in each task in session 3. Once a chip was picked, the experimenter found the corresponding trial and identified the lottery chosen in that trial.

To execute the classical lottery, subjects picked one ping-pong ball out of 100. On each ping-pong ball, we had painted a unique number from 1 to 100 . If, for example, the lottery specified a $5 \%$ chance of winning 2000 NTD or nothing, the subjects would win 2000 if the number was between 1 and 5, and 0 otherwise. To execute the motor lotteries, subjects performed a single pointing movement to the selected motor lottery. Before the single task, subjects could practice hitting targets as many times as they wanted. On average, the subjects practiced for 10 trials.

\section{Analysis of choice data}

Cumulative prospect theory (CPT) is a descriptive model of choice under risk developed by Tversky and Kahneman (1992) as a major upgrade of prospect theory (Kahneman and Tversky, 1979). In CPT, the subjective utility of a monetary outcome is characterized by the value function, whereas the distortion of probability information is modeled by the probability weighting function. Both are modeled as nonlinear transformations of objective quantities. For each subject, we estimated the value function $v(O)$ and the probability weighting function $w(p)$ separately for the classical lottery task and motor lottery task, based on her choice data. The detailed estimation procedure can be found in the study by Wu et al. (2009).

In CPT, the value function describes the subjective utility for monetary outcome $(O)$ with the functional form $v(O)=O^{\alpha}$. The value of $\alpha$ controlled the shape of the value function and the extent of deviation of subjective utility from object amount. When $\alpha=1$, the subjective utility of outcome value is equivalent to objective amount. When $\alpha<1$, the value function is concave, indicating a decrease in marginal utility as the objective amount increases. When $\alpha>1$, the value function is said to be convex, indicating an increase in marginal utility as the objective amount increases.

The probability weighting function captures how the subject used probability to weight subjective utility. Previous studies have shown that people tend to overweight small probabilities and underweight moderate-to-large probabilities in classical lottery tasks in which information about probability was explicitly communicated to the subjects in number or graphic form (Tversky and Kahneman, 1992; Gonzalez and $\mathrm{Wu}, 1999$; Wu et al., 2009). The functional form of the weighting function we chose was a one-parameter form proposed by Prelec (1998): $w(p)=\exp \left[-\left(-(\ln (p))^{\gamma}\right], 0<p<1\right.$.

$\mathrm{CPT}$ is based on the assumptions that the subject assigns a prospect value (PV) to each lottery and that, in comparing lotteries, the subject chooses the lottery with the higher prospect value. For our simple lotteries with only one non-zero outcome, the prospect value is just $w(p) v(O)$.

\section{Imaging data acquisition}

Imaging data were collected with a 3 T MRI whole-body scanner (Siemens Magnetom Tim Trio) equipped with a high-resolution 32-channel head array coil. T2*-weighted functional images were collected using an echo planar imaging sequence (TR, $2 \mathrm{~s}$; TE, $30 \mathrm{~ms}$; 33 axial slices acquired in ascending interleaved order; $3.4 \times 3.4 \times 3.4 \mathrm{~mm}$ isotropic voxel; $64 \times$ 64 matrix in a $220 \mathrm{~mm}$ field of view; flip angle, $90^{\circ}$ ). Each subject completed six scans in one scanning session. There were 24 trials in each scan. Each trial lasted $8 \mathrm{~s}$ with an intertrial interval of $12 \mathrm{~s}$. Consequently, each scan consisted of 240 images. T1-weighted anatomical images were collected after the functional scans using an MPRAGE sequence (TR, $2.53 \mathrm{~s}$; TE, $3.03 \mathrm{~ms}$; flip angle, $7^{\circ}$; 192 sagittal slices; $1 \times 1 \times 1 \mathrm{~mm}$ isotropic voxel; $224 \times 256$ matrix in a $256 \mathrm{~mm}$ field of view).

\section{fMRI statistical analysis}

Functional imaging data were analyzed using FSL [for FMRIB Software Library (in which FMRIB is Functional MRI of the Brain, Oxford University, Oxford, UK)] (Smith et al., 2004). Motion correction was applied using MCFLIRT (Motion Correction using FMRIB's Linear Image Registration Tool) (Jenkinson et al., 2002) and spatial smoothing using a Gaussian kernel of $8 \mathrm{~mm}$ FWHM; high-pass temporal filtering was applied using Gaussian-weighted least-squares straight line fitting with $\sigma=$ $50 \mathrm{~s}$. Registration was performed using a two-step procedure: echo planar images from each scan were first registered to the high-resolution T1weighted structural image in which non-brain structures were removed using BET (for Brain Extract Tool) and were then registered to the standard MNI space using 12-parameter affine transformations.

fMRI statistical analysis was performed using FEAT (for fMRI Expert Analysis Tool). Time series was prewhitened using FILM (FMRIB's Improved Linear Model) with local autocorrelation correction (Woolrich et al., 2001). FILM estimated the autocorrelation structure in the time series and removed it before conducting GLM analysis. The GLM analysis was performed in three steps. First, lower-level FEAT analysis was performed for each scan/block of each subject. Then a fixed-effect (FE) analysis was performed for each subject that combined the lower-level FEAT results from different scans using the summary statistics approach (Beckmann et al., 2003). That is, the parameter estimate $(\beta)$ of each contrast specified in the first-level analysis was treated as data for the FE analysis. Finally, a mixed-effect analysis using FLAME (FMRIB Local Analysis of Mixed Effects) was performed across subjects by taking the FE results from the previous step and treating subjects as a random effect.

\section{General linear models: decision-making session}

For the decision-making session, we described two different GLMs whose results were reported in this paper.

Model 1. During the lottery presentation period (4s), we implemented a dummy regressor that took the value of 1 and 0 elsewhere. In addition, there were two parametric regressors during this period: a regressor for the subjective utility of monetary outcome, $v(O)$, of the varying lottery $(p, \$ O)$ and a regressor for the probability weight, $w(p)$. Note that, because the reference lottery was fixed within a block, there was no need to create $v(O)$ and $w(p)$ regressors for it. During the choice period (2s), there was a dummy regressor having the value of 1 and 0 elsewhere.

Model 2. During the lottery presentation period (4s), we implemented a dummy regressor that took the value of 1 and 0 elsewhere. In addition, the product of the subjective utility of outcome and probability weight (prospect value) constituted the parametric regressor during this period. During the choice period $(2 \mathrm{~s})$, there was a dummy regressor having the value of 1 and 0 elsewhere. 
Note that, because PV is the product of $v(O)$ and $w(p)$, the correlations between PV and $v(O)$ and between PV and $w(p)$ were high under the current lottery design. Hence, a GLM that included $v(O), w(p)$, and PV would very likely make the $\beta$ estimate associated with each of them less efficient and reliable. A more realistic attempt was to implement PV in a different GLM by itself with one caution when interpreting the results. That is, an area that is significantly correlated with $v(O)$ or $w(p)$ (as a result of model 1) does not imply that it is uniquely correlated with $v(O)$ or $w(p)$ but not PV. Similarly, an area significantly correlated with PV obtained from model 2 does not imply that it is only correlated with PV but not $v(O)$ or $w(p)$. Because the main goal of the experiment was to dissociate outcome-related signals [e.g., $v(O)$ ] from probability-related signals [e.g., $w(p)]$ (which is why we want the correlation between outcome and probability to be low) and not to dissociate outcome- or probabilityrelated signals from EV-related signals (e.g., PV), the GLMs implemented here remained suitable to address the main research question.

\section{General linear models: size judgment session}

For the size judgment session, we ran one GLM. During the presentation of the two options, we set up two sets of regressors. The first set of regressors was created to model the trials in which the subjects were asked to judge the size of numbers. The second set of regressors was created to model trials for judgment of target size between the two options. Each set contained three regressors: a dummy regressor that took the value of 1 during the presentation period and 0 elsewhere, a regressor for the number in the varying option, and a regressor for the size of the rectangular target in the varying option. During the choice period ( $2 \mathrm{~s})$, there was a dummy regressor having the value of 1 and 0 elsewhere.

\section{Results}

\section{Behavioral results}

In Figure 3, $A$ and $B$, we plotted the subjects' estimated probability weighting functions separately in the classical lottery task (Fig. $3 A$ ) and in the motor lottery task (Fig. $3 B$ ). Each curve represented the estimated weighting function from a single subject. As can be seen in the graphs, individual subjects exhibited different extent of probability distortion in both the classical and the motor lottery task. In general, we found that the pattern of distortion was in the opposite direction between the classical and the motor tasks, as reported by Wu et al. (2009). That is, subjects tended to overweight small probabilities and underweight moderate-tolarge probabilities in the classical lottery task. The black thick curve in Figure $3 A$ represented the median weighting function in the classical task ( $\alpha=0.8274)$. Conversely, in the motor lottery task, subjects tended to underweight small probabilities and overweight moderate-to-large probabilities, with the black thick curve in Figure $3 B$ representing the median weighting function $(\alpha=1.3315)$.

In Figure $3 C$, we plotted the estimated values of the value function parameter $\alpha$ in the motor lottery task against the classical lottery task. Each data point represented a single subject. For the classical task, 13 of 16 subjects had $\alpha<1$. Compared with the classical task, there were more subjects having $\alpha>1$ in the motor task. In general, there seemed to be a pattern that $\alpha$ was larger in the motor lottery task than in the classical lottery task. In a linear regression analysis in which we regressed $\alpha$ in the motor task against $\alpha$ in the classical task, we found that the estimated slope $\left(\hat{\beta}_{1}\right)$ was significantly different from 1 but not significantly different from $0\left(\hat{\beta}_{1}=0.0943\right)$, suggesting that $\alpha$ was not significantly correlated between the two tasks. The estimated intercept $\left(\hat{\beta}_{0}\right)$ was significantly different from $0\left(\hat{\beta}_{0}=0.9037\right)$, suggesting that $\alpha$ in general was greater in the motor task than the classical task. This outcome differed from that of Wu et al. (2009) in which no difference between motor and classical $\alpha$ was found.

In Figure $3 D$, we plotted the subjects' mean verbal estimates of probability of hitting the motor target as a function of probability
A

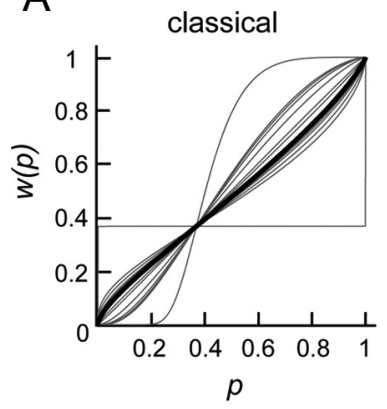

B

C
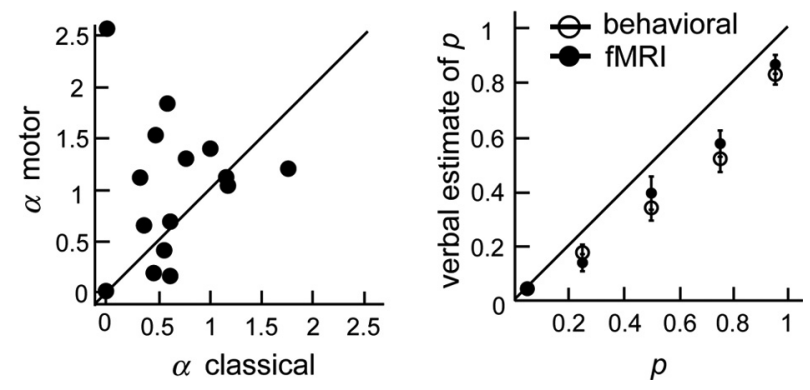

Figure 3. Behavioral results. $\boldsymbol{A}$, Estimated probability weighting functions in the classical lottery task. Each gray curve represented the estimated $w(p)$ of a single subject. The black thick curve indicated the median $w(p)$. $\boldsymbol{B}$, Estimated $w(p)$ in the motor lottery task. Each gray curve represented the estimated $w(p)$ of a single subject. The median $w(p)$ was indicated by the black thick curve. $\boldsymbol{C}$, The estimated parameter value that characterized the shape of the value function in the motor task was plotted against that in the classical task. Each data point represented a single subject. $\boldsymbol{D}$, At the conclusion of the fMRI session, the subjects were presented with five motor targets of different sizes and asked to indicate her verbal estimates on the probability of hitting the target. The subjects gave verbal estimates in both the scanner and the behavioral testing room. The subjects' mean verbal estimate of probability of hit was plotted as a function of probability. The filled dots indicated the verbal estimates obtained while the subjects were in the scanner, whereas the open dots indicated estimates obtained from the behavioral testing room. Error bars represented \pm 1 SEM.

of hit based on her performance in the motor training session. The subjects were asked to give verbal estimates both inside the scanner and in the behavioral testing room. Because the viewing condition inside the scanner was likely different from that in the behavioral testing room, we need to ensure that the ruler implementation was successful (for details, see Materials and Methods) and that the subjects' verbal estimates inside the scanner were consistent with those in the behavioral testing room. We separately plotted the mean verbal estimates obtained from the MRI scanner (color coded in black) and mean estimates obtained from the behavioral testing room (color coded in blue). Error bars represented \pm 1 SEM. At all five levels of probability, we did not find significant difference between the two viewing conditions. The subjects on average had accurate estimates of probability, although there was a slight tendency to underestimate probability.

\section{Neural correlates of the subjective utility of monetary outcome}

We found that activity in the MPFC and the posterior cingulate cortex (PCC) was significantly correlated with the value function, i.e., the subjective utility of monetary outcomes, estimated from the subjects' choice data. Figure $4 A$ showed the results of random-effect analysis of two contrasts at the group level. One contrast was to test parameter estimate $(\beta)$ of the subjective utility of outcome against 0 in the classical task, whereas the other was to test the $\beta$ of the regressor of subjective utility of outcome 
A
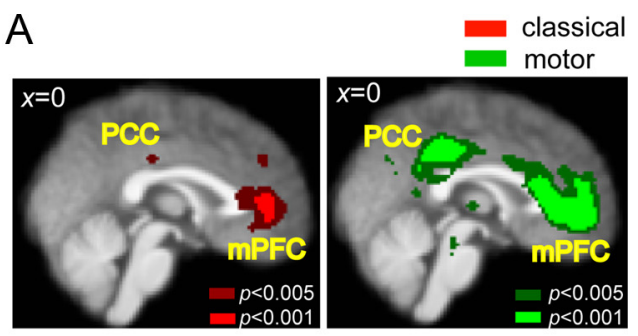

B

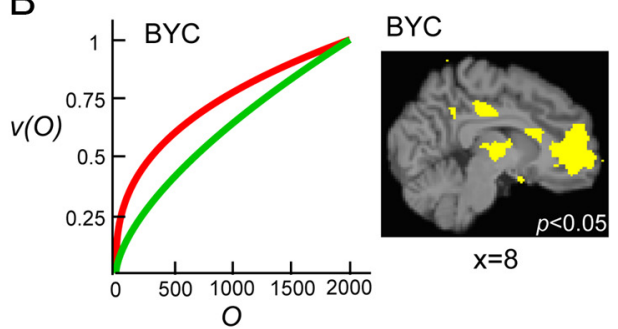

C

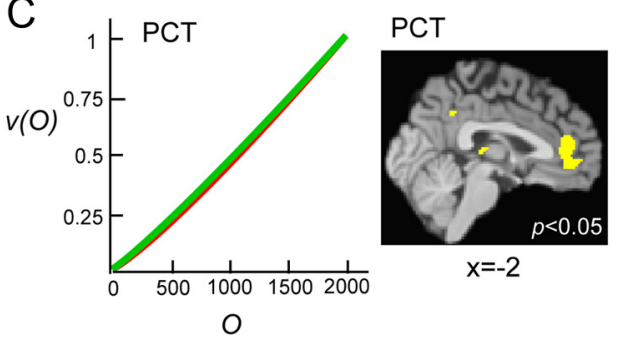

D

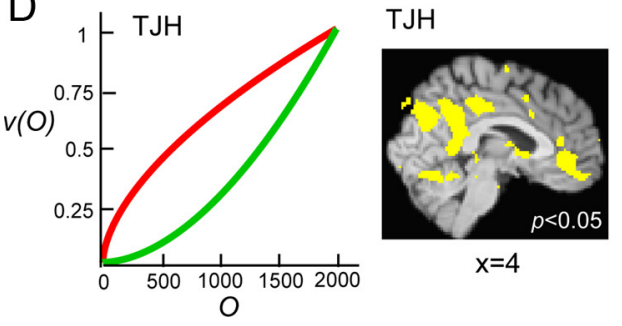

Figure 4. Neural correlates of the subjective utility of monetary outcome. $A$, Statistical parametric maps obtained from random-effect analysis at the group level. Green represented clusters of voxels positively and significantly correlated with the subjective utility of monetary outcome, i.e., the value function in cumulative prospect theory (dark green, $p<0.005$ uncorrected; bright green, $p<0.001$ uncorrected). Red represented clusters of voxels (cluster size $>$ 15 voxels) significantly and positively correlated with the value function in the classical task at the same thresholds. Regions in the $\operatorname{mPFC}[$ classical: peak voxel, $(4,42,10), z=3.6$; motor: peak voxel, $(0,46,-2]), z=3.89$ ] and the PCC [classical: peak voxel, $(16,-52,30), z=3.12$; motor: peak voxel, $(0,-34,40), z=3.73]$ significantly correlated with the value function in both tasks. $\boldsymbol{B}-\boldsymbol{D}$, Results from three individual subjects. For each subject, we plotted the value function, $v(0)$, separately estimated for the classical task (red) and the motor task (green) against objective monetary outcome $(0)$ on the left and areas significantly correlated with $v(0)$ in both tasks (conjunction analysis). The statistical maps were overlaid on the individual subject's high-resolution $\mathrm{T} 1$ image. Each subject exhibited unique shapes of $v(0)$, and activity in $\mathrm{mPFC}$ and PCC represented these distinct shapes that could be seen at the individual level in these subjects. Voxel coordinates were in MNI space.

against 0 in the motor task. The statistical maps for the classical task and the motor task were color coded by red and green, respectively. To further illustrate that MPFC and PCC represent the value functions of subjects having a wide range of parameter estimate, Figure $4 B-D$ showed the value functions estimated from choice behavior for three subjects and the regions in the brain whose activity was correlated with the value functions. For each subject, the left graph showed the estimated value functions (red, classical task; green, motor task); the right column showed common areas correlated with value function in both tasks as a result of a conjunction analysis. From model 1 described above (see Materials and Methods, General linear models: decisionmaking session), at the single-subject level, we obtained two statistical maps, one for the value-function contrast (testing the $\beta$ of the value function regressor against 0 ) in the classical task, and the other for the value-function contrast in the motor task. We then separately thresholded and binarized the two statistical maps at $p<0.05$ (uncorrected). The clusters shown in yellow were the results of multiplying the binarized statistical maps. A similar approach in reporting single-subject results can be seen in the study by Kable and Glimcher (2007). Each subject exhibited a distinct shape of the value function: subject BYC's (Fig. $4 B$ ) value function was concave in both tasks, subject PCT's value function was close to linear in both tasks, and subject TJH's value function was concave in classical but convex in motor task. Such large individual differences are typical of decision-making data (Luce, 2000). Despite the variability in the estimated value functions across subjects and the two tasks, activity in $\mathrm{MPFC}$ and PCC was significantly correlated with the value function.

\section{Neural correlates of probability weight}

Figure $5 A$ showed the results of random-effect analysis of two contrasts at the group level. One contrast was to test parameter estimate $(\beta)$ of the probability-weight regressor against 0 in the classical task, whereas the other was to test the $\beta$ of the probability-weight regressor against 0 in the motor task. Regions significantly correlated with probability weight were displayed with distinct color coding for classical (red) and motor (green) trials.

We found that distinct regions in $\mathrm{mPFC}$ represented probability weight in both the classical and motor lottery tasks. The mPFC activation in the motor task was more ventral and extended to the anterior cingulate cortex compared with the classical task. Conversely, regions in $\mathrm{MPFC}$ correlated with probability weight in the classical task were more in the dorsal part of mPFC. In addition, activity in the visual cortex was correlated with probability weight in the motor lottery task.

Figure $5 B-D$ showed the results from three subjects each having unique shapes of the probability weighting function. For each subject, the left graph showed the probability weighting functions estimated from choice behavior (red, classical task; green, motor task); the right column showed common areas correlated with probability weighting function in both tasks as a result of a conjunction analysis. From model 1 described above (see Materials and Methods, General linear models: decision-making session), at the single-subject level, we obtained two statistical maps, one for the probability-weight contrast (testing the $\beta$ of the probability-weight regressor against 0 ) in the classical task and the other for the probability-weight contrast in the motor task. We then separately thresholded and binarized the two statistical maps at $p<0.05$ (uncorrected). The clusters shown in yellow were the results of multiplying the binarized statistical maps. Each subject exhibited a distinct shape of probability weighting function: subject BYC (Fig. 5B) exhibited the typical shape, i.e., overweighting of small probabilities and underweighting of moderate-to-large probabilities in both tasks; subject $\mathrm{CCH}$ underweight small probabilities and overweight moderate-to-large probabilities in both tasks; and subject TJH's probability weighting function between the two tasks exhibited the opposite direction of distortion. Despite the variability in the shape of the weighting functions, activity in mPFC was significantly correlated with individually estimated weighting function in both tasks, as illustrated in Figure $5 B-D$. 
A

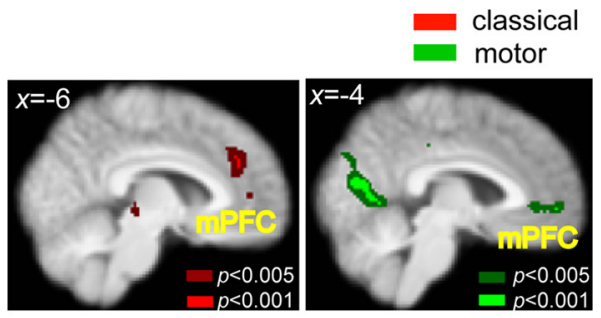

B

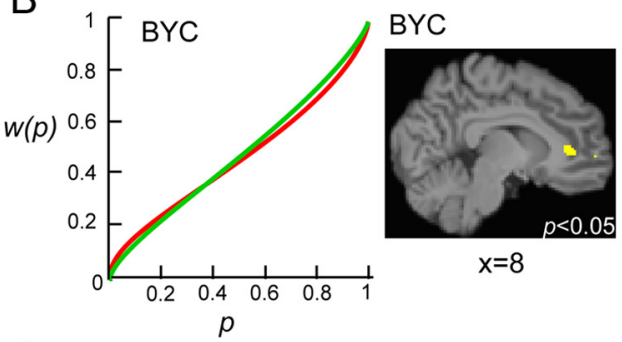

C
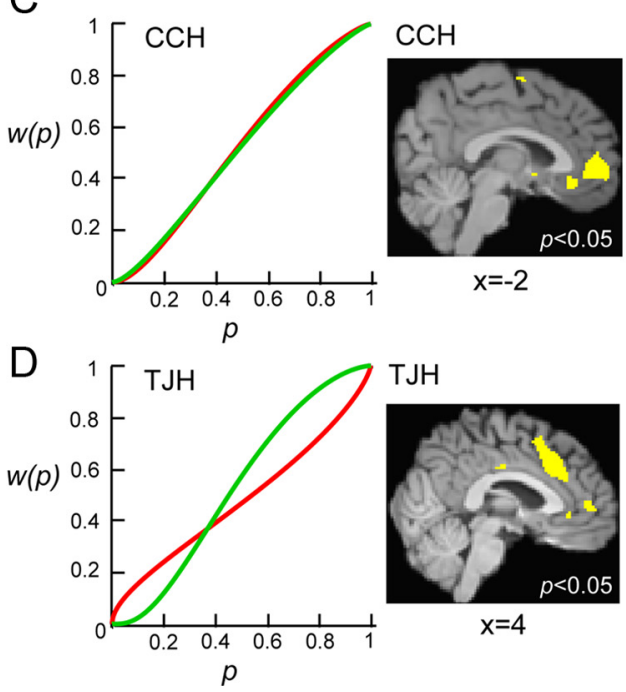

Figure 5. Neural correlates of probability weight. $\boldsymbol{A}$, Statistical parametric maps obtained from random-effect analysis at the group level. Green represented clusters of voxels positively and significantly correlated with probability weight (dark green, $p<0.005$; bright green, $p<$ 0.001 uncorrected). Red represented clusters of voxels (cluster size $>15$ voxels) significantly and positively correlated with probability weight in the classical task at the same thresholds. Distinct regions in the mPFC [classical: peak voxel, $(-8,36,30), z=3.19$; motor: peak voxel, $(-4,54,-2), z=3.12]$ significantly coded for probability weight in both tasks. Activity in the occipital cortex exhibited strong correlation with probability weight in the motor task [peak voxel, $(-18,-54,-10), z=4.22]$. $\boldsymbol{B}-\boldsymbol{D}$, Results from three individual subjects. For each subject, we plotted the probability weighting function, $w(p)$, separately estimated for the classical task (red) and the motor task (green) on the left and areas significantly correlated with the $w(p)$ in both tasks (conjunction analysis). The statistical maps were overlaid on the individual subject's high-resolution $\mathrm{T} 1$ image. Each subject exhibited unique shapes of $w(p)$, and activity in $\mathrm{mPFC}$ represented these distinct shapes that could be seen at the individual level in these subjects. Voxel coordinates were in MNI space.

\section{Neural correlates of prospect value}

We defined the PV of a lottery $(p, \$ O)$ as the product of $v(O)$ and $w(p)$. In Figure 6, we showed regions that were significantly correlated with PV. We found that regions in MPFC (in both the dorsal and ventral parts of $\mathrm{mPFC}$ ) and the lateral orbitofrontal cortex (lOFC) were correlated with PV in the classical task (Fig. $6 A$ ). For the motor task, we found that $\mathrm{mPFC}$ and PCC were correlated with PV (Fig. 6B).

The previous analyses focused on identifying regions in which the correlation between BOLD response and the variables of in- terest, i.e., subjective utility of outcome, probability weight, and prospect value, was significantly different from 0 . The results cannot tell us which areas in the brain are more correlated with these variables of interest in one task than the other or whether the sign of correlation is different between the two tasks. We performed a separate analysis to answer this question. For probability weight, we found that the lingual gyrus in the occipital cortex was the only region for the difference between the motor and the classical tasks (contrast: motor - classical, $p<0.001$ uncorrected), whereas no region was significant at the same threshold for the contrast (classical - motor).

For the subjective utility of monetary outcome, we found that the correlation of activity in the supplementary motor area (SMA) with the (motor - classical) contrast was positive and was marginally significant $[p<0.005$ uncorrected; peak voxel, $(-2$, $-14,50) ; z=3.03$; no voxel was significant at $p<0.001$ uncorrected]; we did not find any region to be positively correlated with the (classical - motor) contrast at the same threshold. Closer inspection on the $\beta$ estimates around the peak voxels in SMA revealed that activity in this region was positively correlated with subjective utility in the motor task but was negatively correlated with it in the classical task. For prospect value, we also did not find any region significantly correlated with the (classical - motor) contrast at $p<0.005$ (uncorrected). However, for the (motor - classical) contrast, we found that a region in the primary motor cortex was significant at $p<0.001$ (uncorrected) [peak voxel, $(-2,-30,80) ; z=3.23]$. The $\beta$ estimate of the prospectvalue contrast in the motor task was positive and was negative for the same contrast in the classical task. The differences in the sign of correlation between the two tasks are potentially interesting. However, it was beyond the scope of the current study to address as to what this might imply.

\section{Comparison between the lottery task and the size judgment task}

In the motor lottery task, information about the probability of obtaining monetary outcome was determined jointly by the size of the motor target and the subjects' own motor noise. Hence, regions whose activity was correlated with probability weight could simply reflect the representation of the physical size of the bar, not probability per se. The size judgment task served to resolve this issue. The stimuli in this task were identical to the motor lottery task. What the subjects were asked to do, however, was different from the motor lottery task. In every trial, two options, each consisting of a number and a bar, were presented. The subjects were asked to compare either the size of bar or the size of number between two options.

We found that mPFC was significantly correlated with probability weight in the motor lottery task but was not correlated with bar size in the size judgment task. This ruled out the possibility that activity in mPFC simply reflects response to the physical size of the bar. The difference between motor task and size judgment task (contrast: motor probability weight - bar size) in $\mathrm{mPFC}$ was also significant. For the same contrast, we also found significant difference in the ventral striatum (vStr) (Fig. $7 B)$ and the lingual gyrus (LG) extending to the temporal occipital fusiform cortex (Harvard-Oxford cortical structural atlas) (Fig. 7C). For vStr, the $\beta$ estimates extracted was positive for the probability-weight contrast in the motor task but was negative for the bar-size contrast in the size judgment task. For LG, the correlation between its activity and probability weight in the motor task and the correlation between activity in LG and bar size in the size judgment task were both positive and significantly different from 
A

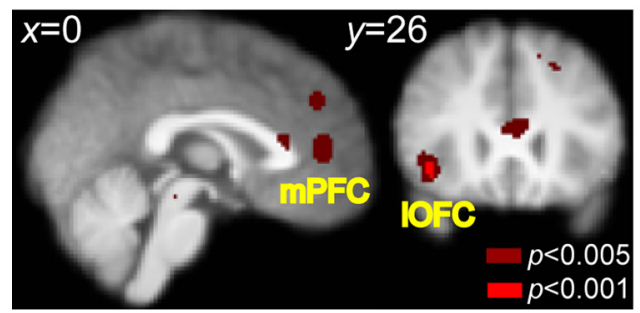

$\mathrm{B}$

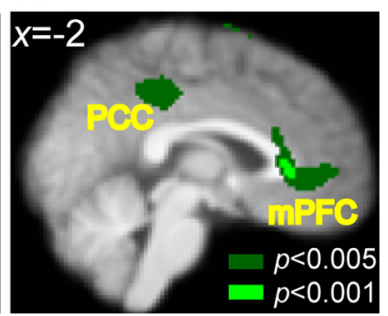

Figure 6. Neural correlates of prospect value. PV of a lottery $(p, \$ 0)$ was defined by the product of the subjective utility of outcome $v(0)$ and its associated probability weight $w(p)$ inferred from choice behavior. $A$, Regions in $\mathrm{mPFC}$ [peak voxel, $(-6,48$, $8), z=3.02$ ] and the right IOFC [peak voxel, $(40,26,-8), z=3.25$ ] were correlated with PV in the classical task (cluster size > 15 voxels; dark red, $p<0.005$ uncorrected; bright red, $p<0.001$ uncorrected). $\boldsymbol{B}$, For the motor task, we found that $\mathrm{mPFC}$ [peak voxel, $(-2,36,-2), z=3.03$ ] and PCC [peak voxel, $(-2,-34,42), z=3.07]$ was significantly correlated with PV at the same thresholds. Voxel coordinates were in MNI space.
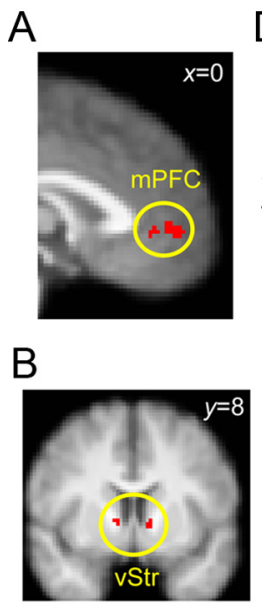

C

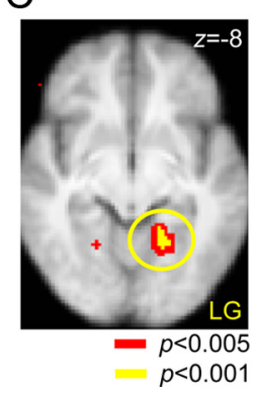

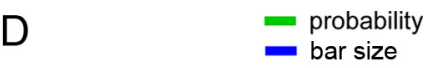

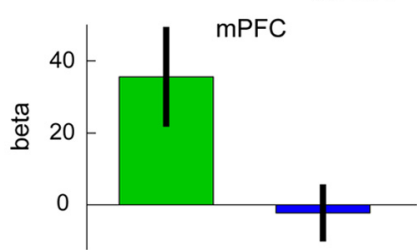

E

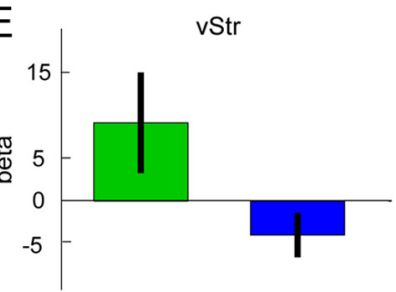

$\mathrm{F}$

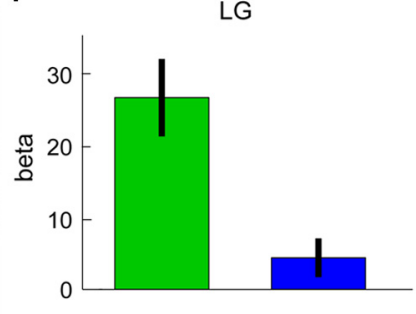

Figure 7. Neural representation of probability weight compared with bar size. We compared the neural representation of probability weight in the motor lottery task with the neural representation of bar size in the size judgment task. $\boldsymbol{A}$-C, Thresholded statistical map for the contrast (probability weight in motor task - bar size in the size judgment task) was shown on the left column in $\mathrm{mPFC}$ [peak voxel, $(-4,54,-2], z=3.05]$, vStr [peak voxel, $(-10,8,0), z=2.77$ ] and the LG in the occipital cortex [peak voxel, $(-18,-52,-8), z=3.30$ ]. Voxel coordinates were in MNI space. $\boldsymbol{D}-\boldsymbol{F}$, Mean parameter estimates (beta) of the probability-weight contrast obtained from the motor lottery task and the size contrast from the size judgement task were extracted from the mPFC, vStr, and LG regions of interest shown in $\mathbf{A - C}$ and plotted. Green represents the probability-weight contrast, while blue represents the size contrast. Error bars, \pm 1 SEM.

0 . LG exhibited significantly stronger correlation with probability weight during motor decision making than with bar size during size judgment.

\section{Discussion}

In this study, we demonstrated that the mPFC represents information about probability in two distinct forms of decision under risk.
Research in this area typically uses lotteries that contain explicit specifications of outcome values and corresponding probabilities (Kahneman and Tversky, 1979, 2000). However, many decision problems we face everyday involves choosing among lotteries, i.e., probability distribution over possible outcomes, in which information about probability is known to the chooser but is not explicitly given. In this study, we investigated the neural correlates of outcome and probability under two different task modalities when subjects were engaged in making risky decisions. The key difference between the two types of lottery choice tasks was how probability information was revealed to the chooser. In the classical lottery task, information about probability was explicitly provided to the subjects. In the motor lottery task, probability was not explicitly given and was dependent on the subjects' own motor noise, noise in executing a rapid pointing movement. On each trial subjects had to estimate their probabilities of obtaining monetary rewards when evaluating the motor lotteries, based presumably on their knowledge of motor noise obtained from the motor training session of the experiment.

We found that the same areas of MPFC and PCC compute the subjective utility of monetary outcome in lotteries at the time of choice regardless of whether probability of winning monetary rewards was described explicitly or learned implicitly through trial and error in a motor task. For information about probability, we found that activity in mPFC and the occipital cortex were significantly correlated with probability weight, i.e., the distortion of information about probability of reward during decision under risk, of obtaining monetary rewards in the motor task. For the classical lottery task, we also found distinct regions in mPFC that quantitatively represented probability weight. The mPFC results on probability in the classical task were in general consistent with previous studies (Knutson et al., 2005; Preuschoff et al., 2006; Yacubian et al., 2006) (for review, see Platt and Huettel, 2008; Rushworth and Behrens, 2008). Past research also has shown that the MPFC is involved in the computation of subjective value of choice object in a wide variety of tasks (Kable and Glimcher, 2007; Plassmann et al., 2007) (for review, see Kable and Glimcher, 2009) and across different types of goods (Chib et al., 2009). We found that activity in the mPFC was correlated with probability weight when it was explicitly revealed to the subjects and when it had to be inferred through previous experience with a motor task. This result indicates that, although probability may be estimated by different neural systems depending on how it is revealed, such modality-specific representation converges to key valuation structures such as the mPFC during decision making. To summarize, our results suggest the possibility of a common representation of information about probability weight in $\mathrm{mPFC}$ when it is explicitly revealed (classical lottery task) and when it needs to be estimated by taking into account motor noise (motor lottery task).

When information about probability of reward was learned from experience in a simple conditioning task in which a certain stimulus was associated with a particular probability of reward, fMRI studies have shown that part of the reward system, such as the striatum (Abler et al., 2006; Tobler et al., 2007, 2008), was correlated with probability. A recent study (Fitzgerald et al., 2010) that compared the neural correlates of value learned from experience and value explicitly described to the subjects showed 
that the ventromedial prefrontal cortex and PCC were positively correlated with learned value, whereas described value was correlated with activity in the ventral putamen. In this study, we found that MPFC was correlated with prospect value in both the classical lottery task and motor lottery task. Note that information about monetary outcome in both tasks was explicitly revealed, whereas information about probability was explicitly revealed in the classical task but had to be estimated based on motor experience in the motor task. Future research needs to directly compare situations in which knowledge of reward probabilities was learned through different types of experience, for example, learning about reward probabilities resulting from perceptual or motor noise versus learning in classical "bandit" lottery tasks.

\section{Previous experience and decision making under risk}

There are two issues related to previous experience in this experimental design. The first issue is the subjects' experience associated with the rapid pointing task. The second issue is subjects' experience associated with decision making under risk, i.e., choosing between lotteries in the lottery tasks.

The process of inferring and computing probability of hitting targets (and thereby winning monetary rewards in the motor lottery task) likely relied on subjects' experience with the rapid pointing task during the motor training period before the fMRI session (for design details, see Materials and Methods). Although subjects did not practice choosing between motor lotteries during this training period, they did practice the rapid pointing task and observe their own success or failure to hit the target on each trial. Hence, one conjecture is that, while comparing the motor lotteries in the fMRI session, the subjects simulated movements based on previous experience with the pointing task to compute the probability of winning. The corresponding neural conjecture would be that regions involved in motor imagery would participate in estimating probability information in the motor task. Our study, however, does not provide evidence that the subjects are simulating the motor task at the time of choice. The results indicated that the correlation between probability weight in the motor task and BOLD response in regions involved in motor imagery, primarily the posterior parietal cortex and the motor cortices, was not significantly different from 0 . When we compared the extent of correlation with probability weight in motor task against that in the classical task, we did find that the resulting parameter estimate was significantly different in the visual cortex and was marginally significant in the posterior parietal cortex and the motor cortices. Although nonsignificant results in fMRI need to be interpreted with caution (Tom et al., 2007), based on the current result, we cannot conclude that the motor imagery network is more correlated with probability in the motor task than in the classical task.

Hertwig et al. (2004) conjectured that choice patterns are different when subjects have acquired probability through experience than when probability is explicitly provided. In their study, however, "experience" referred only to simulated practice with the actual decision task. It is well known that decision performance changes with repeated choices among lotteries (Thaler and Johnson, 1990; Redelmeier and Tversky, 1992; Wakker et al., 1997). Subjects in the present study only practiced the motor task, i.e., repeatedly attempting a single target varying its size and received feedback on hit or miss after each attempt in the training period. They did not receive feedback or gain "experience" in choosing between motor lotteries until the very end of the experiment outside the scanner where they were allowed to execute two of their chosen lotteries, one from the motor and the other from classical.

Hence, although subjects' experience with the motor task during training did play a critical role in establishing the knowledge of his or her motor noise and consequently the likelihood of hitting motor targets, such experience was experience concerning their own motor successes and failures, not experience with the decision task.

\section{References}

Abler B, Walter H, Erk S, Kammerer H, Spitzer M (2006) Prediction error as a linear function of reward probability is coded in human nucleus accumbens. Neuroimage 31:790-795.

Beckmann CF, Jenkinson M, Smith SM (2003) General multilevel linear modeling for group analysis in FMRI. Neuroimage 20:1052-1063.

Berns GS, Capra CM, Chappelow J, Moore S, Noussair C (2008) Nonlinear neurobiological probability weighting functions for aversive outcomes. Neuroimage 39:2047-2057.

Chib VS, Rangel A, Shimojo S, O’Doherty JP (2009) Evidence for a common representation of decision values for dissimilar goods in human ventromedial prefrontal cortex. J Neurosci 29:12315-12320.

Christopoulos GI, Tobler PN, Bossaerts P, Dolan RJ, Schultz W (2009) Neural correlates of value, risk, and risk aversion contributing to decision making under risk. J Neurosci 29:12574-12583.

Churchland MM, Afshar A, Shenoy KV (2006) A central source of movement variability. Neuron 52:1085-1096.

Fitzgerald TH, Seymour B, Bach DR, Dolan RJ (2010) Differentiable neural substrates for learned and described value and risk. Curr Biol 20:1823-1829.

Gonzalez R, Wu G (1999) On the shape of the probability weighting function. Cogn Psychol 38:129-166.

Heekeren HR, Marrett S, Bandettini PA, Ungerleider LG (2004) A general mechanism for perceptual decision-making in the human brain. Nature 431:859-862.

Hertwig R, Barron G, Weber EU, Erev I (2004) Decisions from experience and the effect of rare events in risky choice. Psychol Sci 15:534-539.

Hsu M, Krajbich I, Zhao C, Camerer CF (2009) Neural response to reward anticipation under risk is nonlinear in probabilities. J Neurosci 29:2231-2237.

Huettel SA, Stowe CJ, Gordon EM, Warner BT, Platt ML (2006) Neural signatures of economic preferences for risk and ambiguity. Neuron 49:765-775.

Jenkinson M, Bannister P, Brady M, Smith S (2002) Improved optimization for the robust and accurate linear registration and motion correction of brain images. Neuroimage 17:825-841.

Kable JW, Glimcher PW (2007) The neural correlates of subjective value during intertemporal choice. Nat Neurosci 10:1625-1633.

Kable JW, Glimcher PW (2009) The neurobiology of decision: consensus and controversy. Neuron 63:733-745.

Kahneman D, Tversky A (1979) Prospect theory: an analysis of decision under risk. Econometrica 47:263-291.

Kahneman D, Tversky A (2000) Choices, values, and frames. New York: Cambridge UP.

Knight FH (1921) Risk, uncertainty, and profit. Boston: Houghton and Mifflin.

Knutson B, Taylor J, Kaufman M, Peterson R, Glover G (2005) Distributed neural representation of expected value. J Neurosci 25:4806-4812.

Körding KP, Wolpert DM (2004) Bayesian integration in sensorimotor learning. Nature 427:244-247.

Luce RD (2000) Utility of gains and losses: measurement-theoretical and experimental approaches. London: Erlbaum.

Newsome WT, Britten KH, Movshon JA (1989) Neuronal correlates of a perceptual decision. Nature 341:52-54.

Paulus MP, Frank LR (2006) Anterior cingulate activity modulates nonlinear decision weight function of uncertain prospects. Neuroimage 30:668-677.

Plassmann H, O’Doherty J, Rangel A (2007) Orbitofrontal cortex encodes willingness to pay in everyday economic transactions. J Neurosci 27:9984-9988.

Platt ML, Huettel SA (2008) Riskybusiness: the neuroeconomics of decision making under uncertainty. Nat Neurosci 11:398-403. 
Prelec D (1998) The probability weighting function. Econometrica 66:497-527.

Preuschoff K, Bossaerts P, Quartz SR (2006) Neural differentiation of expected reward and risk in human subcortical structures. Neuron 51:381-390.

Redelmeier DA, Tversky A (1992) On the framing of multiple prospects. Psychol Sci 3:191-193.

Rushworth MF, Behrens TE (2008) Choice, uncertainty ad value in prefrontal and cingulate cortex. Nat Neurosci 11:389-397.

Smith SM, Jenkinson M, Woolrich MW, Beckmann CF, Behrens TEJ, Johansen-Berg H, Bannister PR, De Luca M, Drobnjak I, Flitney DE, Niazy R, Saunders J, Vickers J, Zhang Y, De Stefano N, Brady JM, Matthews PM (2004) Advances in functional and structural MR image analysis and implementation as FSL. Neuroimage [Suppl 1] 23:S208-S219.

Thaler R, Johnson EJ (1990) Gambling with the house money and trying to break even: the effects of prior outcomes on risky choice. Manage Sci 36:643-660

Tobler PN, O’Doherty JP, Dolan RJ, Schultz W (2007) Reward value coding distinct from risk attitude-related uncertainty coding in human reward systems. J Neurophysiol 97:1621-1632.

Tobler PN, Christopoulos GI, O’Doherty JP, Dolan RJ, Schultz W (2008) Neuronal distortions of reward probability without choice. J Neurosci 28:11703-11711.
Tom SM, Fox CR, Trepel C, Poldrack RA (2007) The neural basis of loss aversion in decision-making under risk. Science 315:515-518.

Trommershäuser J, Maloney LT, Landy MS (2003a) Statistical decision theory and tradeoffs in the control of motor response. Spat Vis 16:255-275.

Trommershäuser J, Maloney LT, Landy MS (2003b) Statistical decision theory and the selection of rapid, goal-directed movements. J Opt Soc Am A 20:1419-1433.

Trommershäuser J, Gepshtein S, Maloney LT, Landy MS, Banks MS (2005) Optimal compensation for changes in task-relevant movement variability. J Neurosci 25:7169-7178.

Tversky A, Kahneman D (1992) Advances in prospect theory: cumulative representation of uncertainty. J Risk Uncertain 5:297-323.

Wakker PP, Thaler RH, Tversky A (1997) Probabilistic insurance. J Risk Uncertain 15:7-28.

Woolrich MW, Ripley BD, Brady J, Smith SM (2001) Temporal autocorrelation in univariate linear modeling of fMRI data. Neuroimage 14:1370-1386.

Wu SW, Trommershäuser J, Maloney LT, Landy MS (2006) Limits to human movement planning in tasks with asymmetric gain landscapes. J Vis 6:53-63.

Wu SW, Delgado M, Maloney LT (2009) Economic decision-making compared to an equivalent motor task. Proc Natl Acad Sci U S A 106:6088-6093.

Yacubian J, Gläscher J, Schroeder K, Sommer T, Braus DF, Büchel C (2006) Dissociable systems for gain- and loss-related value predictions and errors of prediction in the human brain. J Neurosci 26:9530-9537. 